Ann. Zootech., x970, 19 (I), 27-36.

\title{
ÉTUDE DE LA SENSIBILITÉ DES CELLULES MYOÉPITHÉLIALES DE LA GLANDE MAMMAIRE DE BREBIS EN FONCTION DES CARACTÉRISTIQUES DE TRAITE
}

\author{
J. LABUSSIÈRE et J. MARTINET \\ avec la collaboration technique de J.-F. COMBAUd, Jacqueline PALY, \\ Micheline Azema et Aline Solari \\ Laboratoire de Physiologie de la Lactation, \\ Centre national de Recherches zootechniques, 78 -Jouy-en-Josas \\ Institut national de la Recherche agronomique
}

\section{RÉSUMÉ}

Au cours de la traite mécanique des brebis, deux types d'animaux se distinguent :

a) ceux fournissant immédiatement le lait citernal, puis environ 30 secondes plus tard, le lait alvéolaire, sous l'effet du réflexe neuro-cndocrinien d'éjection;

b) ceux ne présentant pas cette deuxième émission.

Si cette absence est due à une sensibilité trop faible du myoépithélium à l'ocytocine, nous serons en mesure de le confirmer en comparant in vivo et in vitro la réponse du tissu mammaire de ces deux groupes d'animaux à des quantités connues de l'hormone post-hypophysaire.

\section{In vivo}

Les quantités minima d'ocytocine susceptilles de provoquer une augmentation de pression intramammaire sont identiques pour les cleux lots :

\section{$5,32 \mathrm{mII}$ pour les brebis à 2 pics \\ $5,86 \mathrm{mUI}$ pour les brebis à I pic}

En outre, des doses hormonales croissantes entrainent des effets semblables dans les deux groupes (tabl. I), les droites de régression permettant d'estimer l'élévation de pression en fonction de l'intensité du traitement (log. dose d'ocytocine) étant respectivement de :

$$
\begin{aligned}
& y 2 \text { pics }=9,28 \log x-\mathrm{I} 3,5 \mathrm{I} \\
& y \text { I pic }=9,70 \log x-\mathrm{I}, 6 \mathrm{I} \quad \text { (fig. I } b)
\end{aligned}
$$

$$
\text { 2. In vitro }
$$

Nous arrivons à des conclusions analogues. Les doses seuils provoquant la plus petite contraction des lambeaux de tissu glandulaire sont respectivement de :

l'écart n'étant pas significatif $(\mathrm{P}<0,05)$. 
L'application de l'ocytocine en quantités croissantes n'a pas permis non plus de distinguer de différence entre les deux types de brebis (tabl, 2 et fig. I a). L'amplitude des contractions augmente de façon linéaire avec le log des doses d'ocytocine selon les droites de régression suivantes :

$$
\begin{aligned}
& \text { y } 2 \text { pics }=29,2 \log x-0,869 \\
& y \text { I pic } \quad 27,5 \log x-1,464
\end{aligned}
$$

Tous ces faits tendent donc à prouver que l'absence de la deuxième émission constatée sur certaines courbes de descente du lait, lors de la traite mécanique :

I. n'est pas due à une sensibilité trop faible du récepteur mammaire à l'ocytocine ;

2. résulte d'une perturbation (nerveuse ou endocrinienne) du réflexe d'éjection du lait.

\section{IN'TRODUC'TION}

Certaines brebis de la race Préalpes-du-Sud ne présentent pas, au cours de la traite mécanique, la deuxième émission de lait caractéristique du réflexe neuro-

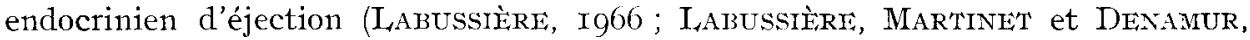
I969). Cette absence peut résulter d'une inhibition partielle ou totale de ce réflexe conduisant à une disponibilité d'ocytocine insuffisante ou même inexistante.

Cependant, il faut également envisager l'éventualité d'une réalisation normale des chaînons nerveux et humoraux dont l'efficacité serait masquée, chez ces animaux à une émission, par une sensibilité trop faible des cellules myoépithéliales.

C'est pourquoi il nous a paru utile de préciser pour les deux types de brebis à un pic et à deux pics :

a) La dose minimum d'ocytocine pouvant provoquer une contraction du myoépithélium ;

b) L'effet, sur ce même tissu, de quantités croissantes de l'hormone post-hypophysaire.

\section{MATÉRIEL E'T MÉTHODES}

Le travail a été réalisé au cours d'essais in vivo et in vitro.

$$
\text { A. - Essais in vivo }
$$

Nous disposions de 35 brebis en première, deuxième et troisième lactation dont 2 I Préalpes$d u$-Sud et $\mathrm{I}_{4}$ issucs d'un croisement to Préalpes $\times$ of ot Frisons. Les courbes de descente de lait effectuées au moment de la traite, aux environs clu $60^{\circ}$ jour de lactation, nous permettent de distinguer 24 animaux à 2 pies et I I à I pic ( $\left.{ }^{1}\right)$.

L'expérience proprement dite commence une semaine après ces contrôles. Elle comporte les opérations suivantes :

1er jour: Mise à jeun et suppression de la traite du soir.

2e jour :

I. Suppression de la traite du matin ;

2. Anesthésie générale de l'animal par une injection intra-jugulaire d’un mélange "starter" contenant $5 \mathrm{ml}$ de pentobarbital sodique à $6 \mathrm{p}$. Ioo (Nembutal Abbott) et $45 \mathrm{ml}$ d'une solution de thiopental sodique à 2 p. Ioo (Pentothal Abbott).

3. Entretien de l'anesthésie par un barbotage d'oxygène et de protoxycle d'azote clans du méthoxyflurane (Penthrane Abbott).

( $\left.{ }^{1}\right)$ Ces types d'animaux ont été décrits d'une façon détaillée au cours de deux études précéclentes : Labussilke (i966), Labussière, Martinet et Devayur (ig69). 
4. Pose d'un cathéter dans le trayon droit et enregistrement de la pression intramammaire de base à l'aide :

- d'un capteur de pression Sanborn type 268 A ;

- d'un enregistreur Sanborn modèle I50 équipé d'un préamplificateur modèle I50-3 ooo.

5. Recherche de la dose minimum d'ocytocine (Syntocinon Sandoz) qui, injectée rapidement ( $\left.{ }^{2}\right)$ par voie intrajugulaire est susceptible de provoquer une élévation de la pression intramammaire puis, mise en évidence de l'effet de doses croissantes de l'hormone post-hypophysaire.

Les trois doses choisies sont sélectionnées en fonction du seuil de sensibilité propre à chaque Brebis. Elles sont calculées pour croître selon une progression logarithmique et administrées toutes les 8 minutes en tenant compte du schéma suivant :

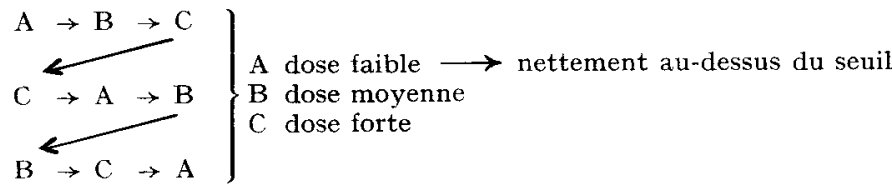

\section{B. - Essais in vitro}

Ils ne portent que sur une partie des brebis utilisées pour le travail in vivo: ro Préalpes et 9 croisées, réparties en 12 animaux à 2 pics et 7 à I pic.

Les essais in vivo et in vitro sont simultanés. Lors de l'anesthésie générale, un lambeau de tissu glandulaire est prélevé sur la moitié gauche de la mamelle en position postéro-interne nettement au-dessus de la zone citernale.

Ce lambeau est lui-même partagé en deux bandelettes dont les caractéristiques sont décrites sur lo tableau suivant:

\begin{tabular}{|c|c|c|c|c|}
\hline & \multicolumn{2}{|c|}{ Brebis à 1 pic } & \multicolumn{2}{|c|}{ Brebis à $\supseteq$ pics } \\
\hline & Moyenne & $\begin{array}{c}\text { Écart-type } \\
\text { de la moyenne }\end{array}$ & Moyenne & $\begin{array}{l}\text { Iicart-type } \\
\text { de la moyenne }\end{array}$ \\
\hline $\begin{array}{l}\text { Poids (mg) des bande- } \\
\text { lettes } \ldots \ldots \ldots \ldots \ldots \ldots . \\
\text { longueur }(m m) \text { des ban- } \\
\text { delettes } \ldots \ldots \ldots \ldots \ldots\end{array}$ & $\begin{array}{r}254,5 \\
20,7\end{array}$ & $\begin{array}{r}13,9 \\
1,2\end{array}$ & $\begin{array}{r}217,2 \\
21,9\end{array}$ & $\begin{array}{r}25,7 \\
1,8\end{array}$ \\
\hline
\end{tabular}

Chaque bandelette est placée dans une cuve à organe isolé. Elle est assujettie :

- par son extrémité inférieure à un crochet solidaire de la cuve ;

- par son extrémité supérieure à une jauge de contrainte (Transducer Sanborn FTA r).

La cuve est alors remplie d'une solution saline dans laquelle on entretient un bulle à bulle d'un mélange d'oxygène et de gaz carbonique dans les proportions respectives de $95 \mathrm{p}$. 100 , 5 p. roo. La solution saline présente la composition suivante (en g/roo $\mathrm{ml}$ ) :

\begin{tabular}{|c|c|c|c|}
\hline $\mathrm{NaCl} \ldots \ldots \ldots \ldots \ldots$ & 0,8 & $\mathrm{Ca} \mathrm{Cl} \mathrm{Cl}_{2} \ldots$ & $0,02 \mathbf{2}^{\prime}$ \\
\hline $\mathrm{K} \mathrm{Cl} \ldots \ldots \ldots \ldots \ldots$ & 0,084 & $\mathrm{NaHCO}_{3}$ & 0,05 \\
\hline $\mathrm{Mg} \mathrm{Cl}_{2} \ldots \ldots \ldots \ldots$ & 0,010 & Glucose ........ & 0,05 \\
\hline
\end{tabular}

(MARTinet et Lis, 1969)

(2) Moins de 5 secondes. 
Le volume du bain est de 3,5 millilitres. La température est maintenue à $3^{\circ} \mathrm{C}$.

Après 15 minutes d'attente, la tension de chaque bandelette est réglée à I 50 mg, grâce à un étalonnage interne à l'enregistreur (Sanborn, modèle I 50 ; préamplificateur I $50-3$ ooo).

On est alors en mesure de déterminer la dose minimum d'ocytocine (Syntocinon Sandoz) qui, ajoutée au sérum physiologique, est susceptible de provoquer une contraction décelable du tissu mammaire. Comme dans le cas de l'essai in vivo et selon le même protocole expérimental, on enregistre ensuite les contractions de la bandelette de tissu mammaire sous l'effet de quantités croissantes d'ocytocine. Les trois doses choisies ont des valeurs nettement plus élevées que celles du seuil de sensibilité.

I.es traitements sont appliqués toutes les ro minutes et séparés par 3 rinçages de la cuve.

\section{RÉSULTATS}

\section{A. - Expériences in vivo}

I. Pression intra-mammaire de base.

Nouts n'avons pas détecté pour ce critère de différences significatives entre les deux types d'animaux.

\begin{tabular}{|c|c|c|}
\hline & $\begin{array}{c}\text { Pression } \\
\text { intra-mammaire } \\
\text { (en mur Hg) }\end{array}$ & $\begin{array}{c}\text { Écart-type } \\
\text { de la moyenne }\end{array}$ \\
\hline Brebis à 2 pics & 7,99 & 0,10 \\
\hline Brebis à 1 pic $\ldots . . .$. & 7,81 & 0,49 \\
\hline
\end{tabular}

\section{Dose minimum d'ocytocine provoquant une augmentation de pression.}

Le seuil de réponse est de $5,32 \mathrm{mUI}$ pour les brebis à 2 pics et $5,86 \mathrm{mUI}$ pour celles à I pic. La différence n'est pas significative (écart-type de la moyenne respectivement 0,88 et $\mathrm{I}, 03$ ).

La relation entre le seuil de sensibilité et la pression intra-mammaire préexistante n'est pas significative $(r-0,306,35$ couples de données $)$, mais il importe de remarquer que l'accumulation du lait pendant 24 heures dans la mamelle ne correspond pas aux conditions normales de traite.

\section{Effets de quantités croissantes d'ocytocine sur la pression intramammaire.}

Les trois doses injectées varient sensiblement d'un individu à l'autre, les extrêmes étant respectivement de Io mUI, $20 \mathrm{mUI}$, $40 \mathrm{mUI}$ d'une part et de $80 \mathrm{mUI}$, roo mUI, I25 mUI d'autre part. Toutefois, comme l'indique le tableau I, la moyenne des doses faibles pour les brebis à deux émissions ne diffère pas significativement de celle des brebis à une émission. Il en est de même pour les traitements moyens et forts.

C'est pourquoi à l'intérieur de chaque dose nous avons comparé les pressions maximum obtentes pour les deux types d'animaux. Il ressort du tableau I que celles-ci sont très voisines. 


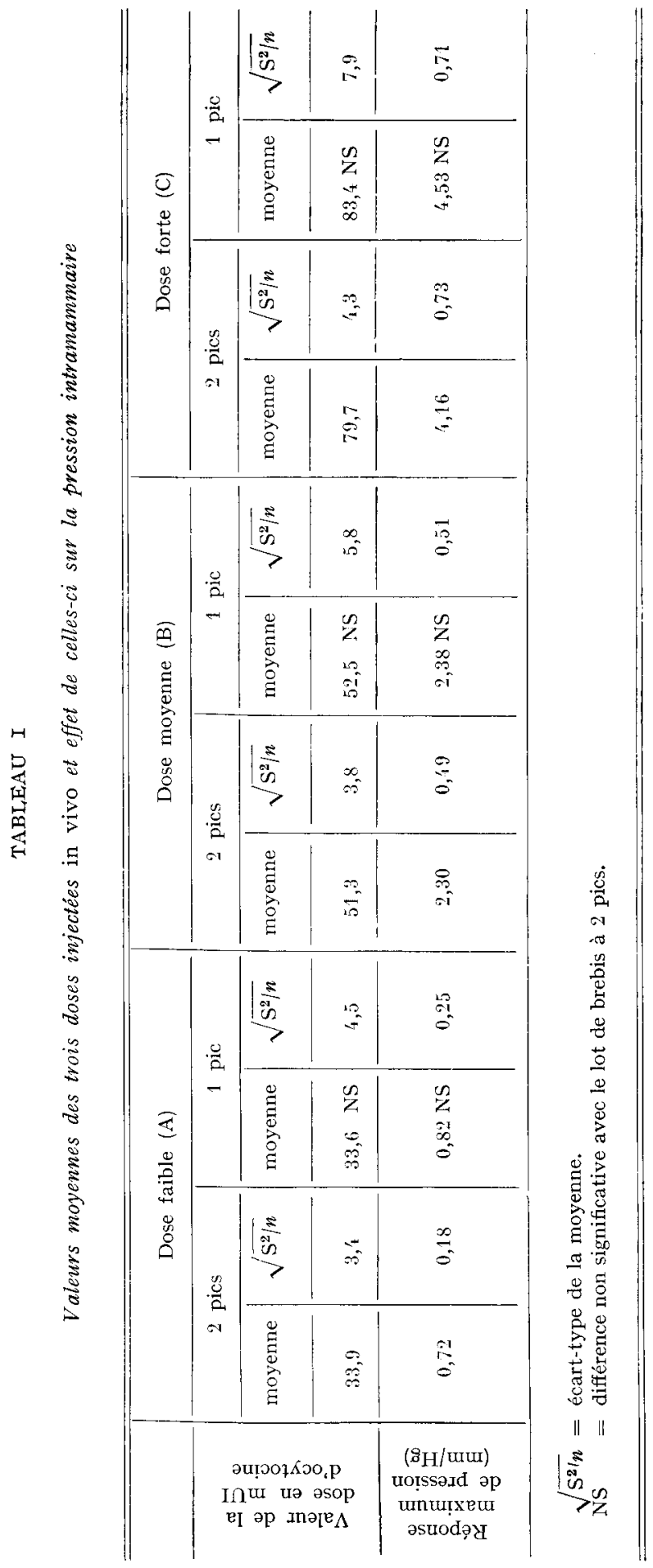




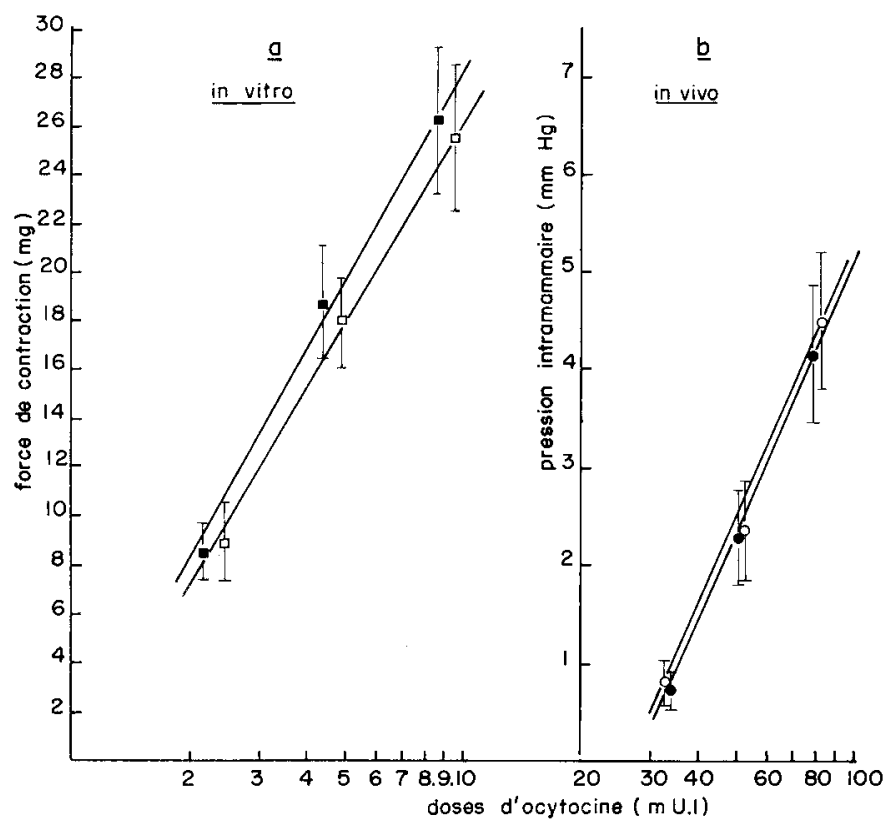

FIG. I. - Effet de quantités croissantes d'ocytocine sur la réponse du tissu mammaire
I $\mathrm{I}$ pic in vitro
- 2 pics in vitro
$O$ I pic in vivo
- 2 pics in vizo

La similitude des droites de régression, construites à partir de ce tableau (fig. I $b$ ) illustre aussi ces résultats.

$$
\begin{aligned}
& y 2 \text { pics }=9,28 \log x-\mathrm{I} 3,5 \mathrm{I} \\
& y \text { I pic }=9,40 \log x-\text { I } 3,6 \text { I } \\
& \text { B. - Expériences in vitro }
\end{aligned}
$$

I. Doses minimum d'ocytocine provoquant une contraction du tissu mammaire.

I,es valeurs seuil obtenues sont beaucoup plus faibles que celles relevées lors de

\begin{tabular}{|c|c|c|}
\hline & $\begin{array}{c}\text { Moyenne } \\
\text { des doses seuil }\end{array}$ & $\begin{array}{l}\text { Écart-type } \\
\text { de la moyenne }\end{array}$ \\
\hline Brebis à 2 pics & $0,171 \mathrm{mUI}$ & 0,038 \\
\hline Brebis à 1 pic $\ldots$. & $0,2 \cdot 2 \cdot 2 \mathrm{mUI}$ & 0,101 \\
\hline
\end{tabular}
l'expérimentation in vivo. Mais, comme précédemment, nous ne pouvons mettre en évidence une différence entre les deux groupes de brebis.

Il n'existe pas de relation significative entre les seuils de réponse in vivo et in vitro $(r=-0,372 ; 12$ couples de données). 


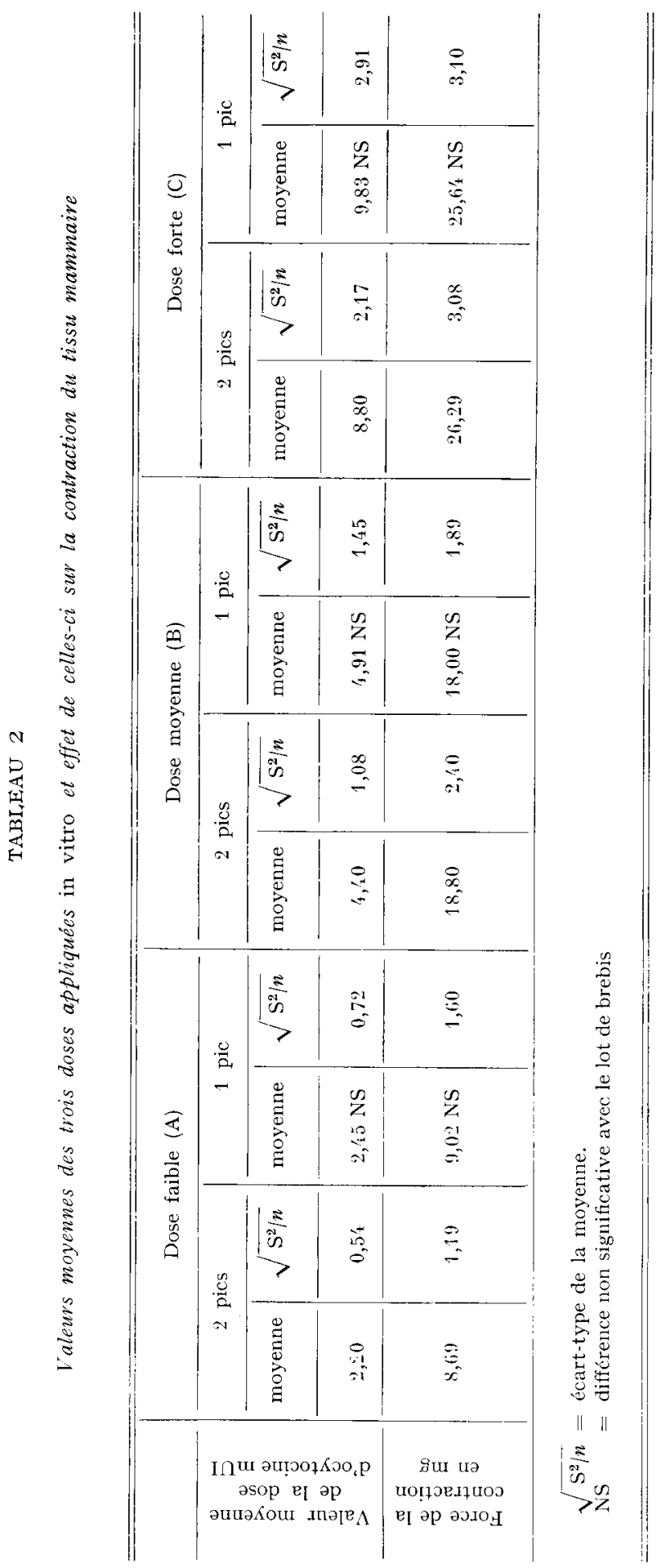


2. Effets de doses croissantes d'ocytocine.

Le tableau 2 et la figure $\mathrm{I}$ a nous indiquent qu'en moyenne :

a) les doses administrées sont sensiblement analogues dans les deux lots, bien qu'il existe de grandes différences individuelles;

b) les bandelettes mammaires de chaque lot se contractent avec la même amplitude lorsqu'on applique la même quantité moyenne d'ocytocine.

La fonction, réponse-logarithme de la dose, est linéaire, les deux droites de régression ayant pour équation :

$$
\begin{array}{ll}
\text { animaux à } 2 \text { pics } & y=29,24 \log x-0,869 \\
\text { animaux à I pic } & y=27,52 \log x-1,464
\end{array}
$$

\section{DISCUSSION}

\section{A. - Senil de sensibilité}

Les doses minima d'ocytocine produisant une éjection du lait in vivo sont du même ordre que celles obtenues par ANDERson (I95I) chez la Brebis et la Chèvre, par Cowie (I952) et par DENAMur et MARTINET (I953) chez la Chèvre.

Les doses seuils sont beaucoup plus faibles dans 1'expérience in vitro que dans celle in vivo.

Deux hypothèses permettent d'expliquer ce phénomène :

I. La première est due au fait que la prise de pression intra-mammaire traduit un phénomène global qui dépend bien entendu de la sensibilité du myoépithélium, mais aussi de critères que nous n'avons pu évaluer, tels que : le rapport des volumes citernaux et alvéolaires, la taille, l'élasticité et la répartition du système canaliculaire.

2. La seconde résulte d'un effet de dilution et de dispersion, dî̀ à la longueur du circuit sanguin entre la veine jugulaire et la glande mammaire.

LINZELL (I955) et LEVITSKAYA (I955) ont effectivement montré chez la Souris et le Cobaye que l'injection d'ocytocine dans l'artère mammaire ou l'application locale, permettent de détecter des valeurs seuils beancoup plus basses que par injection intraveineuse. Toutefois, la quantité minimum agissant au niveau des cellules myoépithéliales reste sensiblement la même (voir revue DENAmur, rg65).

Nous pouvons conclure :

a) qu'il n'existe pas de différence entre les seuils de sensibilité des brebis à une et à deux émissions ;

b) que nous n'avons pu établir de concordance entre les réponses in vivo et in vitro.

\section{B. - Effet de doses croissantes d'ocytocine}

Les recherches concernant l'influence de quantités croissantes d'ocytocine sur l'amplitude de pression intramammaire ont été analysées d'une façon critique par Martinet et Denamur (ig60) et Denamur (I965).

Il en résulte qu'il existe, à l'intérieur de certaines limites, une relation significative linéaire entre la pression intramammaire et le logarithme de la dose d'ocytocine injectée. Nos résultats confirment cette relation. Toutefois, aussi bien in vivo qu'in vitro, il ne nous a pas été possible de différencier les droites de réponses mammaires des deux groupes d'animaux. 
Ces observations tendent donc à prouver que l'absence de la deuxième émission, constatée lors de l'enregistrement des courbes de descente de lait, chez 20 à 30 p. Ioo des brebis Préalpes-du-Sud, n'est probablement pas due à une sensibilité trop faible du récepteur mammaire à l'ocytocine. Sans négliger 1'influence possible de certains facteurs morphologiques (taille et emplacement des trayons, volume de la citerne), pouvant masquer l'existence du réflexe d'éjection du lait, il semble probable que dans de tels cas il y ait, soit interruption de la voie nerveuse, soit absence de libération d'ocytocine (ou libération insuffisante) pour des raisons qu'il serait souhaitable de pouvoir déterminer rapidement.

Rę̧u pour publication en janvier 1970.

\section{REMERCIEMENTS}

Nous tenons à remercier l'ensemble du personnel de la Ferme expérimentale de Brouessy dont l'aide nous a été précieuse pour la réalisation de ce travail.

\section{SUMMARY}

\section{MYOEPITHLLIAI, CEI, SLNSITIVITY IN THE EWE MAMMARY GI,ANI) AS RI'I,ATIS,} TO MILKING CHARAC'TERISTICS

Two types of ewes may be distinguished during mechanical milking :

a) those giving cisternal milk immediately, and then alveolar milk $3^{\circ}$ seconds later in response to the neuroendocrine ejection reflex.

b) those not having the second emission. If this lack is due to a low myoepithelium sensitivity to oxytocin, it can be confirmed by comparing the in vivo and in vitro mammary tissue res. ponse of these two groups to known amounts of the post-pituitary hormone.

\section{In vivo.}

The minimum amounts of oxytocin causing intramammary pressure increase are idcntical for the two lots :

$$
\begin{aligned}
& 5.32 \mathrm{mUI} \text { for 2-peak cwes } \\
& 5.86 \mathrm{mUI} \text { for I-peak ewes }
\end{aligned}
$$

Moreover, increasing hormonal doses has the same effect on the two groups (tabl. I). Pressure rise varies with the intensity of treatment (log oxytocin dose), and is measured by the following regression lines :

2. In vitro.

$$
\begin{aligned}
& \text { y } 2 \text { peaks }=9.28 \log x-\mathrm{I} 3.5 \mathrm{I} \\
& y \text { I peak }=9.40 \log x-\mathbf{I} 3.6 \mathrm{I} \text { (fig. I } b \text { ) }
\end{aligned}
$$

The conclusions obtained are the same as those mentioned above : threshold doses causing the smallest contraction of glandular tissue slices are:

$$
\begin{aligned}
& 0.17 \mathrm{I} \text { mU of oxytocin in 2-peak animals } \\
& 0.222 \mathrm{mUI} \text { of oxytocin in I-peak animals }
\end{aligned}
$$

respectvely. The difference is not significant $(\mathrm{P}<0,05)$.

The difference between the two types of ewes was not better distinguished when increasing amounts of oxytocin were administered (table 2 and fig. $1 a$ ). Contraction amplitude increases in a linear way with the $\log$ of oxytocin doses according to the following regression lines :

$$
\begin{aligned}
& y 2 \text { peaks }=29,2 \log x-0,869 \\
& y \text { I peak }=27,5 \log x-\mathrm{I}, 464
\end{aligned}
$$

These facts tend to prove that absence of the second emission observed in some milk letdown curves during mechanical milking: 
I. is not due to a low sensitivity of the mammary receptor to oxytocin.

2. is the result of a disturbance (nervous or endocrine) of the milk ejection reflex.

\section{RÉFÉRENCES BIBLIOGRAPHIQUES}

Anderson B., r95I. Some observations on the neuro hormonal regulation of milk-ejection. Acta Physiol. Scand., 23, I-7.

Denamur R., Martinet J., r953. Sensibilité de la glande mammaire de la Chèvre aux hormones posthypophysaires. C.R. Soc. Biol., 147, I2 I7-1220.

Dexamur R., 1965. The hypothalamo-neurohypophysial system and the milk ejection reflex. Dairy Sci. Abst, 27, 193-224. Dairy Sci. Abstr., 27, 263-280.

LABUSSière J., I966. Relations entre le niveau de production laitière des brebis et leur aptitude à la traite. XVII Congr. Int. Laiterie, Munich, Section $\mathrm{A}_{1}, 43-5 \mathrm{I}$.

Labussiere J., Martinet J., Denamur R., i969. The influence of the milk ejection reflex on the flow rate during the milking of ewes. J. Dairy Res., 36, I9 I-202.

Levitskaya E. S., I955. Étude in vivo de l'appareil d'éjection du lait de la glande-mammaire de la Souris blanche. Trud. Inst. Fiziol. I. P. Pavlov, 4, 58-62.

LINzELL J. L., 1955. Some observations on the contractile tisste of the mamtnary gland. J. Physiol., $130,257-267$.

Martinet J., Denamur R., I960. Étude préliminaire des mécanismes de l'évacuation du lait de la glande manmaire chez la Chèvre et la Brebis. Arch. Sci. physiol., 14, 35-96.

Martinet J., Lis M., I969. Pharmacological study of the activity of mammary myoepithelium in the guinea pig (à paraître). 